Spontaneous Pattern Formation on Ion Bombarded Si(001), accepted to PRL, 1999

\title{
Spontaneous Pattern Formation on Ion Bombarded Si(001)
}

\author{
Jonah Erlebacher, Michael J. Aziz \\ Harvard University, Division of Engineering and Applied Sciences, Cambridge, MA
}

Eric Chason, Michael B. Sinclair, Jerrold A. Floro

Sandia National Laboratories, Albuquerque, NM

\begin{abstract}
Pattern formation on surfaces undergoing low-energy ion bombardment is a common phenomenon. Here, a recently developed in situ spectroscopic light scattering technique was used to monitor periodic ripple evolution on $\mathrm{Si}(001)$ during $\mathrm{Ar}^{+}$sputtering. Analysis of the rippling kinetics indicates that under high flux sputtering at low temperatures the concentration of mobile species on the surface is saturated, and, surprisingly, is both temperature and ion flux independent. This is due to an effect of ion collision cascades on the concentration of mobile species. This new understanding of surface dynamics during sputtering allowed us to measure straightforwardly the activation energy for atomic migration on the surface to be $1.2 \pm 0.1 \mathrm{eV}$. The technique is generalizable to any material, including high temperature and insulating materials for which surface migration energies are notoriously difficult to measure.
\end{abstract}

PACS numbers: 68.35.Bs, 81.10.Aj, 85.40.Ux 


\section{DISCLAIMER}

\section{Portions of this document may be illegible in electronic image products. Images are produced from the best available original document.}


Spontaneous Pattern Formation on Ion Bombarded Si(001), accepted to PRL, 1999

Low energy $(500-1000 \mathrm{eV})$ ion bombardment is a common technique used in many thin film applications such as forming shallow junctions, sputter etching and deposition, ion beam assisted growth, reactive ion etching, and plasma assisted chemical vapor deposition. Under certain conditions, ion sputtering is known to produce patterns on surfaces. Features such as ripples, bumps or cones are common $[1,2,3]$. Typical length scales of these features are of order 10-1000 nm. In some cases, these features are nuisances, such as in sample thinning for transmission microscopy or depth profiling by secondary ion mass spectroscopy. However these nano-scale patterns also hold promise in applications as varied as optical devices, templates for liquid crystal orientation, and strain-free patterned substrates for heteroepitaxial growth of quantum dots or wires. Rippling has been observed in amorphous materials $\left(\mathrm{SiO}_{2}[1]\right)$, metals $(\mathrm{Ag}[4])$, and semiconductors (Ge [5], Si [6], and others).

In this study, a recently developed in situ light scattering spectroscopic technique [7] was used to monitor ripple evolution on $\mathrm{Si}(001)$ during $\mathrm{Ar}^{+}$sputtering. The technique allowed the first systematic study of the temporal and spatial evolution of both ripple wavelength and ripple amplitude as functions of both temperature and ion beam flux. Theoretical models describe rippling as arising from competition between ion beam roughening/etching and surface diffusion or viscous flow mediated relaxation [8]. Our results here help illuminate the range of validity of these models. Additionally, we describe the method by which the activation energy for surface migration can be found. A salient feature of these experiments was our use of a very high ion flux compared to other rippling experiments. At high fluxes, the annihilation process for mobile species becomes dominated by collision cascades. Sputter-induced rippling can, in principle, occur on the surface of any material. As such, it holds great promise as a method for measuring the activation energy for surface migration on high temperature or insulating materials, which are generally notoriously difficult to measure.

Rippling experiments were performed on $\mathrm{Si}(001)$ (p-type, 3-10 $\Omega-\mathrm{cm}$ ) in an ultrahigh vacuum chamber (base pressure of $2 \times 10^{-10}$ Torr). lons bombarded the surface at glancing incidence, $67.5^{\circ}$ from normal, with energy of $750 \mathrm{eV}$; the projected ion beam 
ran along the [110] direction [9]. Previous studies of sputter rippling have used in situ Xray scattering $[1,2]$, which measured the rms roughness averaged over the surface, or $e x$ situ microscopy [4]. We used a recently developed in situ UV spectroscopic technique, Light Scattering Spectroscopy (LiSSp), that measures the scattered light spectrum in a fixed direction [7]. A broad-band $150 \mathrm{~W}$ Xe arc lamp illuminated the surface during sputtering; a solid state spectrometer collected non-specular scattered light. Fiber optics were used to bring light to and from chamber viewports. The spectrometer response was sufficient to measure spatial features between $200 \mathrm{~nm}$ and $2000 \mathrm{~nm}$, depending on the relative orientation of the illumination and collection optics. For each measured spectrum, a background spectrum without Xe illumination was also measured. This had a dual purpose: (1) subtracting effects of thermal radiation from the UV scattered spectrum and (2) use as a temperature measurement [10].

LiSSp has major advantages over the other methods. The entire scattered light spectrum is acquired, so diffraction peak positions, widths and amplitudes were simultaneously obtained for all relevant feature sizes. Also, by using spectroscopic detection, the need to rotate or move the sample during the measurements is removed. For these reasons, data collection was significantly simplified and a wider range of parameters could be studied. The power spectral density (PSD) of the sample surface (i.e., the square of the magnitude of the Fourier transform of the surface profile, or, equivalently, the surface height-height autocorrelation function) was extracted directly from the measured scattered light spectrum following the analysis in ref [11].

To illustrate the effectiveness of the technique, Figure 1 shows the PSD of a rippled sample, collected in situ at $555^{\circ} \mathrm{C}$. The PSD determined from light scattering spectrum is overlayed with the PSD as measured ex situ using AFM (square symbols)[12]. The peak in the PSD corresponds to a ripple wavelength of $360 \mathrm{~nm}$ and an amplitude of $11 \mathrm{~nm}$, illustrating the sensitivity of the technique. The AFM micrograph of the surface showing the ripples is displayed in the figure inset.

For the ion current of $0.7 \mathrm{~mA} / \mathrm{cm}^{2}$, rippling was monitored over the temperature range $500-600^{\circ} \mathrm{C}$. Figure 2 shows PSD evolution for a representative example. The projected 
Spontaneous Pattern Formation on Ion Bombarded Si(001), accepted to PRL, 1999

directions of the ion beam and scattering vector for the optical spectroscopy were perpendicular to each other, as shown in Figure 1. In the direction parallel to the projected ion beam no significant roughening was observed [13]. The spectra shown were collected in situ at intervals of $500 \mathrm{sec}$. Three features can be seen in Fig. 2, and were generally observed for all data: (1) For large spatial frequencies (above $\sim 0.02 \mathrm{~nm}^{-1}$ in Fig. 2), roughness does not increase, (2) roughness grows at all spatial frequencies below this cut-off, (3) there is a fastest growing spatial frequency, $q^{*}$, whose value is independent of time and dominates all other frequencies at long times. The ripple wavelength, $\lambda^{*}\left(570 \mathrm{~nm}\right.$ in Fig. 2), is simply $2 \pi / q^{*}$.

The foundation of most theories of ion rippling is due to Bradley and Harper (BH) [8]. Theirs is a linear stability analysis of a surface undergoing roughening due to sputtering on one hand and annealing by surface diffusion on the other. Here we summarize the salient points. Consider an ion beam impinging on a surface at an angle $\theta$, measured from normal. A theory of sputter yield due to Sigmund [14] leads to a roughening term that is proportional to the curvature of the surface in the direction perpendicular to the ion beam, $\kappa$. For annealing, mass transport via surface diffusion leads to a relaxation term proportional to the second spatial derivative of $x$ [15]. In the moving reference frame of the sputtered surface, the change in average height with time, $\partial h / \partial t$, can be written

$$
\frac{\partial h}{\partial t}=S \kappa+B \frac{\partial^{2}}{\partial s^{2}} \kappa
$$

where the derivatives are taken with respect to surface arc length, $s$, measured perpendicular to the beam. Under the near-glancing conditions used here, the roughening prefactor is given by $S=-(f a / n) Y_{0}(\theta) \Gamma_{2}(\theta)$, where $f$ is the ion flux, $a$ is the average depth of ion energy deposition, $n$ is the atomic volumetric density, $Y_{0}(\theta)$ is the (incidence angle-dependent) sputter yield, and $\Gamma_{2}(\theta)$ is a coefficient governing the erosion rate dependence on the local surface curvature. The relaxation prefactor is given by $B=D_{s} C \gamma / n^{2} k_{B} T$, where $\gamma$ is the surface free energy per area, $T$ is the temperature, $k_{B}$ 
is Boltzmann's constant, $D_{s}$ is the surface diffusivity, and $C$ is the concentration (number/area) of mobile species that participate in surface diffusion. Eq. (1) is generally valid when $a \kappa<1$, which was always satisfied in our experiments.

In the limit of short times and small slopes (infinitesimal amplitudes), one makes the approximation $\kappa \approx \partial h^{2} / \partial x^{2}$. In this case, Eq. (1) can be solved by Fourier techniques. Specifically, each component, $h_{q}(t)$, of the surface spatial frequency spectrum grows exponentially with rate constant $R_{q}=S q^{2}-B q^{4}$. There is a maximal value of $R_{q}$, indicating that there is a fastest growing spatial frequency, $q^{*}$, independent of time and given by

$$
q^{*}=(S / 2 B)^{1 / 2}
$$

These conclusions are consistent with our observations (1)-(3) above.

Figure $3 \mathrm{~A}$ shows the dependence of the ripple wavelength on temperature at fixed ion flux. The only unknown in Eq. (2) defining $q^{*}$ is the product $D_{s} C$, contained in $B$ [16]. Using the Arrhenius form, $D_{\mathrm{s}} C=A \exp \left(-\varepsilon / k_{B} T\right)$, we obtain a good fit of the data to Eq. (2) with best fit parameter values $A=3.5 \times 10^{10} \mathrm{sec}^{-1}$ and $\varepsilon=1.2 \pm 0.1 \mathrm{eV}$. The data are shown in the figure as open squares and the fit as a line.

If $D_{s}$ and $C$ are both thermally activated and $C$ is equal to its value in equilibrium, then $\varepsilon$ would represent the sum of a migration energy for diffusion, $E_{M}$, and a creation free energy for the diffusing species, $E_{F}$. This sum can be measured by thermal annealing experiments in which lithographically patterned rippled surfaces are heated and their amplitude decay is monitored. For experiments on Si(001), Keeffe, et al [17], find $E_{M}+E_{F}=2.4 \mathrm{eV}$, significantly larger than our measured value for $\varepsilon$. In fact, our measured value $\varepsilon=1.2 \pm 0.1 \mathrm{eV}$ is identical, within experimental error, to the migration energy, $E_{M}=1.1 \pm 0.1 \mathrm{eV}$, for diffusion of dimers on Si(001) measured by STM [18]. A recent measurement by Tromp, et al., indicates that $E_{F}=0.35 \mathrm{eV}$ [19], which is interesting in its inconsistency with the combination of the Keeffe and STM results, but is still so large that numerical comparison of our value with the others makes sense only if 
we interpret $\varepsilon$ as a migration energy alone. This fact suggests that $C$ in our experiment is not thermally activated at all. Furthermore, Tromp, et al., show that the mobile species on the $\mathrm{Si}(001)$ surface at the temperatures relevant here are dimers and not single adatoms, supporting the idea that $\varepsilon$ is the activation energy for surface diffusion of a dimer. For these reasons, we refer to the mobile species as the addimer.

The steady-state concentration of addimers is governed by a balance of their creation and annihilation rates. In the simplest picture, addimers are created at a rate equal to $f Y_{1}$, where $f$ is the ion flux and $Y_{1}$ is the number of addimers created per incident ion. Usually, only annihilation mechanisms involving thermally activated diffusion are considered to be operative. These can be either addimer-addimer aggregation into stable islands or addimer diffusion to traps such as step edges or surface vacancies. A previously unconsidered third possibility is that addimers are directly removed by impinging ions. This process is athermal and the annihilation rate is equal to $f C \sigma_{C}$, where the cross-section $\sigma_{\mathrm{C}}$ is related to the area at the surface over which an ion's damage is deposited in the collision cascade. An impinging ion resets a surface patch of area $\sigma_{C}$ to an addimer concentration that is independent of $T$ and its value before the collision.

We write the net creation rate of addimers as

$$
d C / d t=f Y_{1}-f C \sigma_{\mathrm{C}}-C / \tau_{1}-2 C^{2} / \tau_{2}
$$

where $\tau_{1}$ is the characteristic time for an addimer to diffuse to a step or other surface trap and $\tau_{2}$ is the characteristic time for two addimers to impinge. If the annihilation rate is dominated by direct removal by ion collision cascades, then the steady state concentration is $C_{s s}=Y_{1} / \sigma_{\mathrm{c}}$, independent of $f$ and $T$, and leads via Eq. (2) to $\lambda^{*} \propto f^{-1 / 2}$. For addimer-addimer dominated kinetics, Eq. (3) becomes $d C / d t=f Y_{1}-(2 C)\left(4 D_{s} / a_{0}^{2}\right)\left(C / C_{\text {site }}\right) \sigma_{1}$, where $a_{0}$ is the surface lattice parameter, $C_{\text {site }}$ is the concentration of surface sites, and $\sigma_{1}$ is a dimensionless capture cross-section (the socalled monomer capture number). Identifying $\tau_{2}=a_{0}^{2} C_{\text {site }} / 4 D_{s} \sigma_{1}$ yields $C_{s s} \propto \sqrt{f / D_{s}}$, 
which in turn leads to $B(T) \propto \gamma \sqrt{f D_{s}} / k_{B} T$ and $\lambda^{*} \propto f^{-1 / 4}$. For trap-dominated kinetics, $C_{s s}=f Y_{1} \tau_{1}$. The temperature dependence of $\tau_{1}$ is clarified by writing it in terms of a characteristic length between traps, $l_{\text {trap }}=\sqrt{D_{s} \tau_{1}}$. In steady-state, $l_{\text {trap }}$ does not change with time. This implies $C_{s s} \approx\left(f / D_{s}\right) l_{\text {rrap }}^{2}$, which in turn leads to $B(T) \approx f \gamma / k_{B} T$ and a flux-independent ripple wavelength.

To summarize, the differing scenarios for the steady-state concentration of adatoms yield the following variations of ripple wavelength with flux and temperature:

(a) Ion cascade annihilation:

(b) Addimer-addimer annihilation:

(c) Surface trap annihilation:

$$
\begin{aligned}
& \lambda_{1}{ }^{*} \approx f^{-1 / 2}(k T / \gamma)^{-1 / 2} \exp \left(-E_{M} / 2 k_{B} T\right) \\
& \lambda_{2}{ }^{*} \approx f^{-1 / 4}(k T / \gamma)^{-1 / 2} \exp \left(-E_{M} / 4 k_{B} T\right) \\
& \lambda_{3}{ }^{*} \approx(k T / \gamma)^{-1 / 2}
\end{aligned}
$$

It follows that the dominant annihilation mechanism is identified by measuring the flux dependence of the ripple wavelength.

The positive slope of the data shown in Figure 3A dismisses case (c). Cases (a) and (b) have the same temperature dependence, of the form $(k T)^{-1 / 2} \exp \left(-E / k_{B} T\right)$, but different flux dependencies. We have measured $\lambda *$ at different fluxes, but also at different temperatures. However, we can scale out the temperature dependencies of case (a) and (b) by multiplying the $\lambda^{*}$ values obtained at the various temperatures by $(k T)^{-1 / 2} \exp \left(-E_{M} / 2 k_{B} T\right)$ for case (a) and by $(k T)^{-1 / 2} \exp \left(-E_{M} / 4 k_{B} T\right)$ for case (b).

Figure 3B shows the flux dependence of the critical wavelength as a result of this scaling. Case (a), $\lambda^{*} \propto f^{-1 / 2}$, is the only one consistent with the data and we conclude that we have reached a steady state concentration of mobile species that is both flux and temperature independent. This result is surprising, and is contrary to the usual picture of sputtering, in which the number of surface defects such as addimers increases with flux [20]. Plugging in reasonable values into Eq. (3) for all relevant quantities also supports this conclusion. We do not claim that the dynamic structure of a surface undergoing ion bombardment is always temperature and flux independent. We suggest, rather, that at sufficiently long time at sufficiently high flux or low temperature, the mobile species 
concentration saturates at a value that depends only on the character of the ion/material interaction, i.e., the parameters $Y_{1}$ and $\sigma_{\mathrm{c}}$. This prediction can be further tested by repeating the experiment with differing ion energies.

An underlying assumption of the $\mathrm{BH}$ theory is that the surface energy does not change with local surface orientation, an anisotropy that will tend to suppress, or at least strongly modify, ripple instabilities. For amorphous solids this assumption is reasonable, but for monocrystalline Si well below the thermodynamic roughening temperature, it is somewhat surprising that our results follow BH so well. One possibility is that this effect is negligible for small amplitude to wavelength ratios. Another possibility is that conditions of high addimer supersaturation effectively reduce surface energy anisotropy, thereby making it possible to observe roughening instabilities in nominally anisotropic systems, as was recently suggested by Jesson, et al. [21], using a result from Nozieres and Gallet [22].

In summary, we have characterized the temperature and ion beam flux dependence of the sputter rippling phenomenon on $\mathrm{Si}(001)$. The flux dependence of the ripple wavelength, in particular, leads us to conclude that under high flux ion bombardment the steady-state concentration of mobile species on the surface is flux and temperature independent. This previously unobserved result means the dominant annihilation process of mobile surface species is direct removal by ion collision cascades. This process may play an important role in governing film quality during ion-assisted epitaxial growth of thin films. It also permits a straightforward measurement of the surface migration energy, which for $\mathrm{Si}(001)$ we find to be $1.2 \pm 0.1 \mathrm{eV}$ over the temperature range of $500-$ $650^{\circ} \mathrm{C}$.

The authors acknowledge stimulating discussions with Sean Hearne and Craig Arnold, and technical advice given by John Hunter. This work was supported by DEFG08-89ER45401. Portions of this work were performed at Sandia National Laboratories, supported by the United States Department of Energy under contract DEAC04-94AL85000. 
[1] T. M. Mayer, E. Chason, A. J. Howard, J. Appl. Phys 76, 1633 (1994).

[2] M. V. Ramana Murty et al., Phys. Rev. Lett. 80, 4713 (1998).

[3] G. W. Lewis, et al., Nuc. Inst. and Meth. 170, 363 (1980).

[4] S. Rusponi, C. Baragno, U. Valbusa, Phys. Rev. Lett. 78, 2795 (1997).

[5] E. Chason, et al., Phys. Rev. Lett. 72, 3040 (1994).

[6] G. Carter and V. Vishnyakov, Phys. Rev. B 54, 17647 (1996). J. Erlebacher and M. J. Aziz, Mater Res. Soc. Symp. Proc. 440 (1997). Z. X. Jiang and P. F. A. Alkemade, Appl. Phys. Lett. 73, 315 (1998).

[7] E. Chason, M. B. Sinclair, J. A. Floro, J. A. Hunter, R. Q. Hwang, Appl. Phys. Lett. 72, 3276 (1998).

[8] R. M. Bradley, J. M. E. Harper, J. Vac. Sci. Tech. A 6, 2390 (1988). R. Cuerno, A.L. Barabasi, Phys. Rev. Lett. 74, 4746 (1995). M. A. Makeev, A.-L. Barabasi, Appl. Phys. Lett. 71, 2800 (1997).

[9] The ion beam operated at $1.9 \times 10^{-5}$ Torr. Incident ion fluxes of non-neutrals ranged from 0.2 to $0.7 \mathrm{~mA} / \mathrm{cm}^{2}$. Temperature was maintained by a combination of backside and resistive heating. Careful sample cleaning to remove hydrocarbon contamination was essential for reproducibility. In order to eliminate contamination over the long run times from non-sample sources (such as clips to the heater or reflected sputtering off the chamber walls), samples were apertured using silicon wafers. The effect of the sputtering in this geometry was to cut a wide, deep groove in the sample, of order $1 \mathrm{~cm}$ wide and 10-100 $\mu \mathrm{m}$ deep. Ripples developed in this groove, and could be seen by the naked eye as a diffraction grating.

[10] The spectrometer was a 600 lines $/ \mathrm{mm}$ grating blazed at $400 \mathrm{~nm}$ and a CCD array. The incident light spectrum was found by measuring scattering from a Lambertian diffuse scatterer with uniform reflectivity over the measured spectral range. The peak of the blackbody spectrum was calibrated against an optical pyrometer and thermocouples to provide accurate temperature measurements. 
Spontaneous Pattern Formation on Ion Bombarded Si(001), accepted to PRL, 1999

[11] J. M. Elson, Phys. Rev. B 30, 5460 (1984).

[12] We found no significant change in the light scattering spectra upon quenching the sample from the experimental temperature to room temperature and upon subsequent exposure to atmosphere.

[13] The temperatures at which these experiments were performed are high enough that amorphization does not occur.

[14] P. Sigmund, J. Mater. Sci 8, 1545 (1973).

[15] W. W. Mullins, J. Appl. Phys. 30, 1 (1959). C. Herring, in The Physics of Powder Metallurgy, W. E. Kingston, Ed. (McGraw-Hill, 1951), chap. 8.

[16] Values for all other parameters are $n=0.05$ atoms $/ \AA^{3}, a=29 \AA, Y_{0}\left(\theta=675^{\circ}\right)=$ 0.92 atoms $/$ ion, $\Gamma_{2}\left(\theta=675^{\circ}\right)=-0.0634, \gamma=1.23 \mathrm{~J} / \mathrm{m}^{2}$.

[17] M. E. Keeffe, C. C. Umbach, J. M. Blakely, J. Phys. Chem. Solids 55, 965 (1994).

[18] M. Krueger, B. Borovsky, E. Ganz, Surf. Sci. 385, 146 (1997). B. S. Swartzentruber, Phys. Rev. Lett. 76, 459 (1996). B. S. Swartzentruber, A. P. Smith, H. Johnson, Phys. Rev. Lett. 77, 2518 (1996).

[19] R. M. Tromp and M. Mankos, Phys. Rev. Lett. 81, 1050 (1998).

[20] J. Y. Cavaille and M. Dreschler, Surf. Sci. 75, 342 (1978).

[21] D. E. Jesson, K. M. Chen, S. J. Pennycook, T. Thundat, and R. J. Warmack, J. Electron. Mater. 26, 1039 (1997).

[22] P. Nozieres and F. Gallet, J. Physique 48, 353 (1987). 


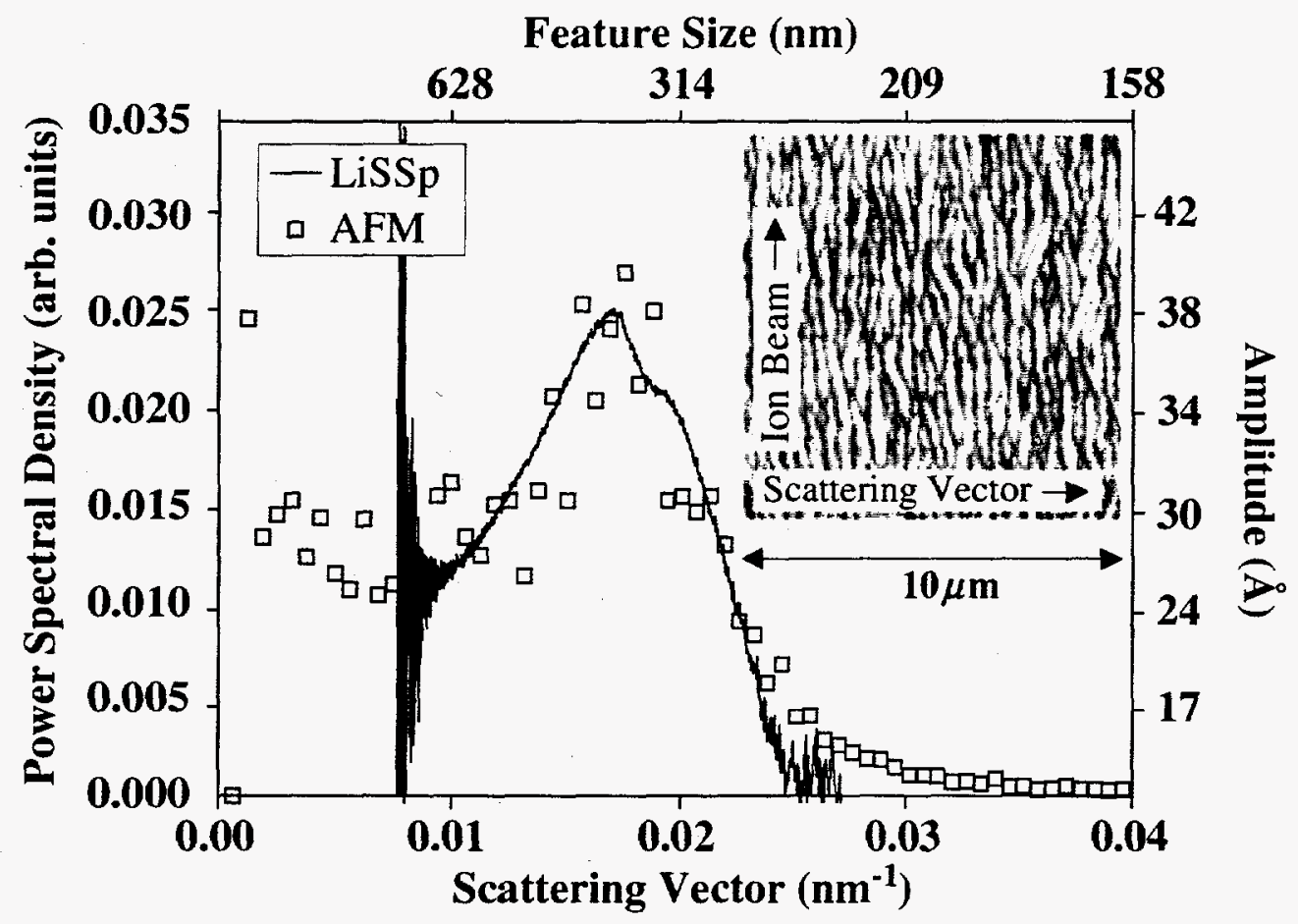

Figure 1. Comparison of the power spectral density as measured by AFM (open squares) and by light scattering. The sample was made by glancing ion beam sputtering $\left(0.7 \mathrm{~mA} / \mathrm{cm}^{2}\right.$ at $67.5^{\circ}$ from normal) at a sample temperature of $555^{\circ} \mathrm{C}$ for 8500 sec. The small discrepancy near $0.025 \mathrm{~nm}^{-1}$ is due to both AFM pixelation error, and spectrometer cutoff. Inset: AFM topograph of this sample showing the projected ion beam direction and the direction of light scattering for the optical spectroscopy. 


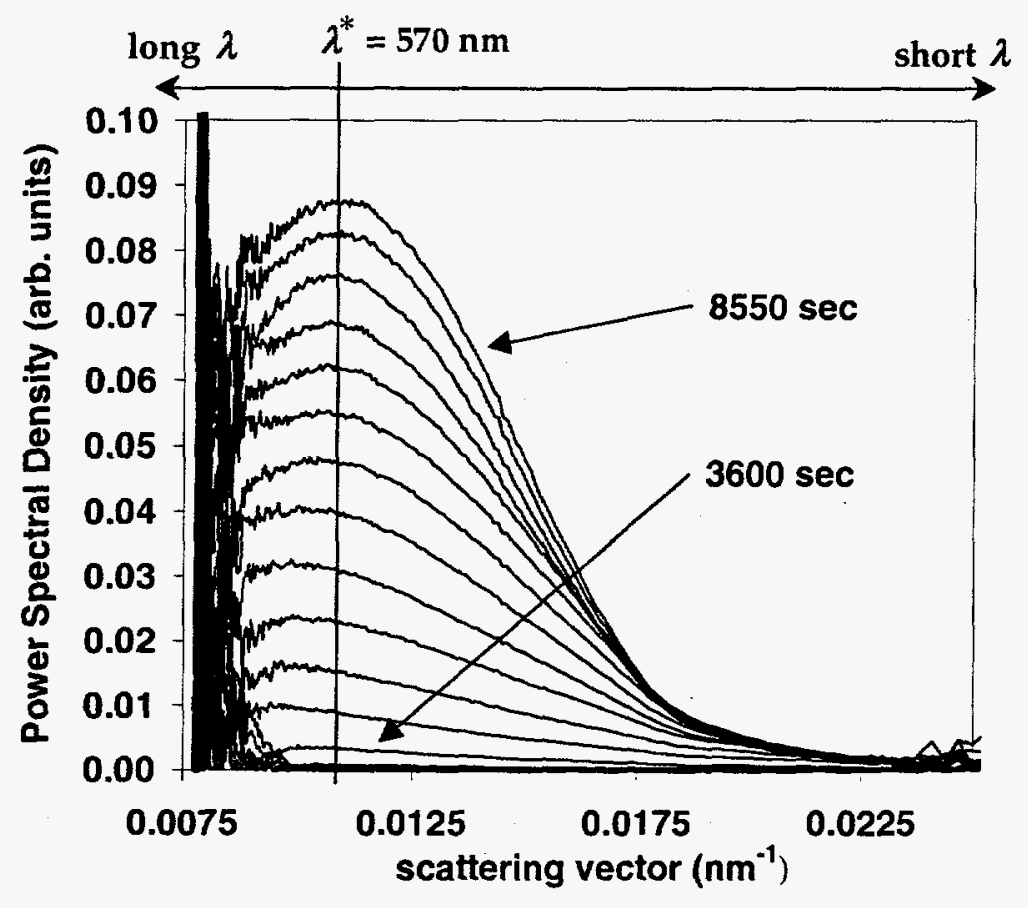

Figure 2. Example of the temporal evolution of the ripple morphology: $T=582^{\circ} \mathrm{C}, \lambda *=570 \mathrm{~nm}$. Curves are measured PSD at intervals of approximately $500 \mathrm{sec}$. The PSD of the fastest growing frequency is not necessarily the greatest at early times due to a non-uniform initial roughness spectrum. 


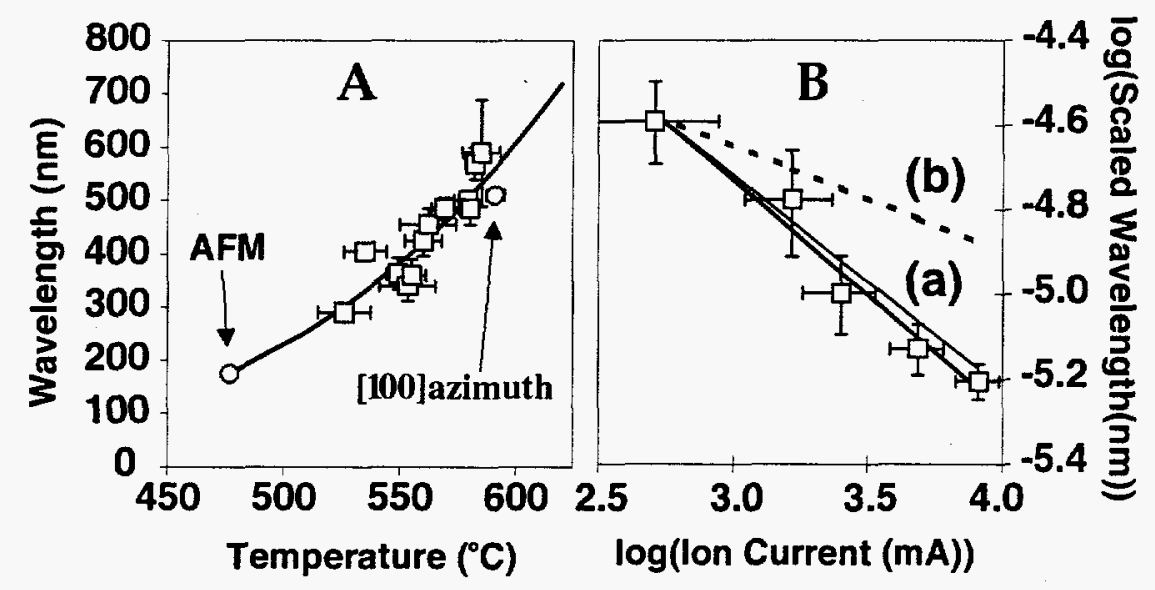

Figure 3. (A) Measured critical wavelength, $\lambda *$, vs. sample temperature (squares), and fit to the prediction of Bradley-Harper theory (line), (B) Scaled critical wavelength vs. ion beam current (out of gun): data (squares); thick line: power law fit, $\lambda^{*} \propto f^{-0.54}$; (a), thin line: power law, $\lambda^{*} \propto f^{-1 / 2}$; (b), dotted line: power law, $\lambda^{*} \propto f^{-1 / 4}$. 


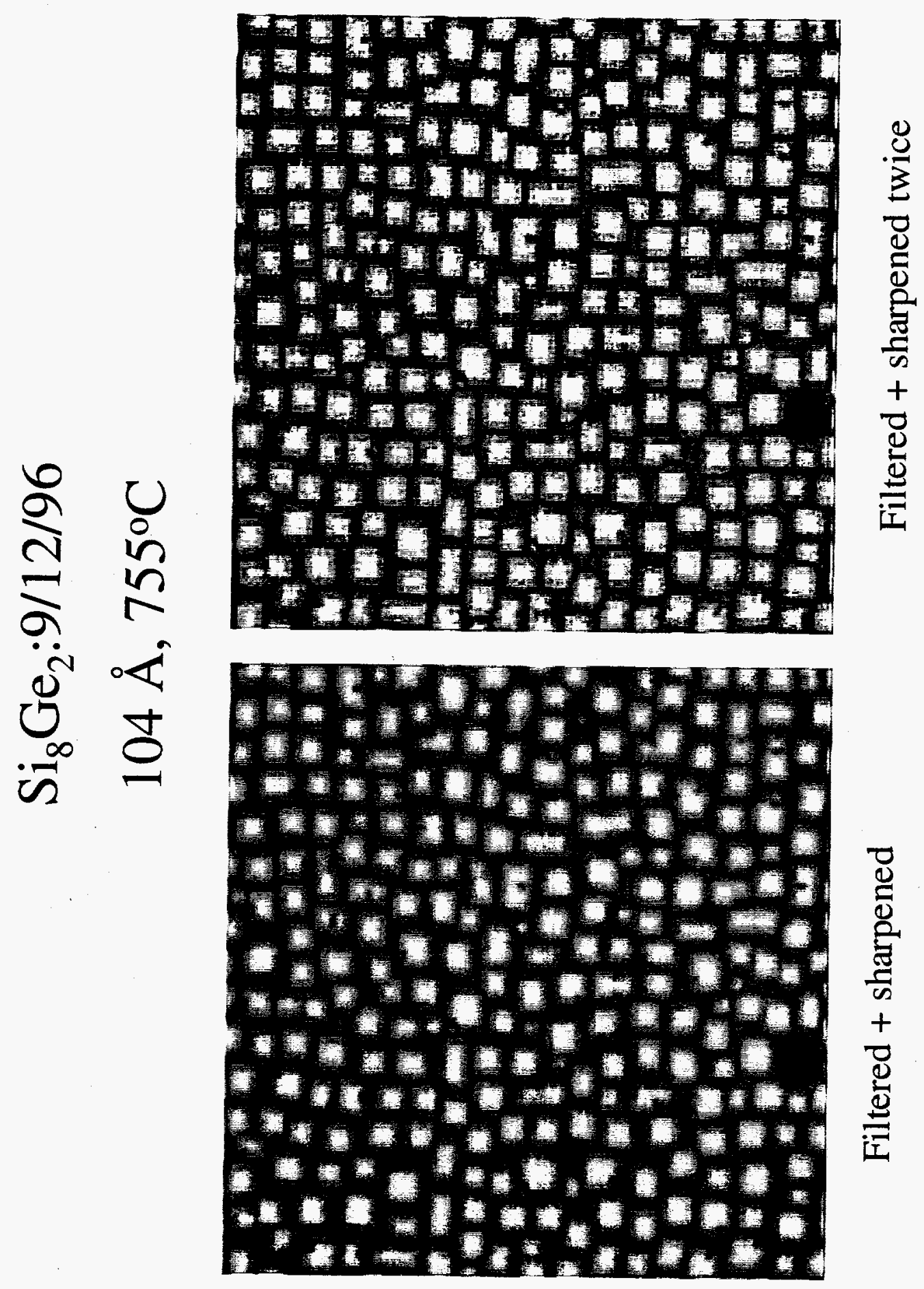

